\title{
Scattering of spin current injected in $\operatorname{Pd}(001)$
}

\author{
J. Foros \\ Simon Fraser University, 8888 University Drive, Burnaby, British Columbia, V5A 1S6, Canada \\ and Department of Physics, Norwegian University of Science and Technology, 7491 Trondheim, Norway \\ G. Woltersdorf and B. Heinrich ${ }^{\text {a) }}$ \\ Simon Fraser University, 8888 University Drive, Burnaby, British Columbia, V5A 1S6, Canada \\ A. Brataas \\ Department of Physics, Norwegian University of Science and Technology, 7491 Trondheim, Norway
}

(Presented on 9 November 2004; published online 28 April 2005)

We have studied spin pumping in $\mathrm{Pd} / \mathrm{Fe}(001)$ ultrathin crystalline films prepared on $\mathrm{GaAs}(001)$ by ferromagnetic resonance (FMR). FMR measurements show that the $\operatorname{Pd}(001)$ overlayers lead to an appreciable attenuation of the spin current, which was generated by the precessing magnetization of Fe. Pd overlayers thicker than about $10 \mathrm{~nm}$ act as perfect spin sinks. It is argued that the loss of spin coherence in Pd is caused by scattering with spin fluctuations. (C) 2005 American Institute of Physics. [DOI: 10.1063/1.1853131]

\section{INTRODUCTION}

Tserkovnyak et al. ${ }^{1}$ showed that a precessing magnetization can generate a spin current into an adjacent normalmetal (NM) layer. The pumped spin current at the interface between the ferromagnetic (FM) layer and NM is given by

$$
\mathbf{j}_{\text {spin }}=\frac{\hbar}{4 \pi} g^{\uparrow \downarrow} \mathbf{n} \times \frac{\partial \mathbf{n}}{\partial t},
$$

where $\mathbf{n}$ is the unit vector along the magnetic moment $\mathbf{M}$, and $g^{\uparrow \downarrow}$ is the interface mixing conductance per unit area in units of $e^{2} / h{ }^{1}$. For interfaces with some degree of diffuse scattering $g^{\uparrow \downarrow}$ is close to the number of transverse channels in $\mathrm{NM}, \Sigma_{m, n} \delta_{m, n}$, see Refs. $2-4$. In simple metals with a spherical Fermi surface this sum is given by

$$
g^{\uparrow \downarrow}=\frac{k_{F}^{2}}{4 \pi} \approx 0.85\left(\frac{N}{2}\right)^{2 / 3},
$$

where $k_{F}$ is the Fermi wave vector and $N$ is the density of electrons in NM. Equation (2) is valid in the limit that the mean free path in the NM film is larger than its thickness. In magnetic double layers FM1/NM/FM2 the spin current injected by FM1 into NM can be absorbed by the ferromagnetic layer FM2. The transverse component of the spin current in NM is entirely absorbed at the NM/FM2 interface. ${ }^{5,6}$ Consequently, the spin current results in an interface Gilbertlike damping for the ferromagnetic layer FM1. For small precessional angles the spin current $\mathbf{j}_{\text {spin }}$ is almost entirely transverse. For good spin sinks, the Gilbert damping is given by the conservation of the total spin momentum and is equal to

$$
\alpha=\gamma \hbar \frac{g^{\uparrow \downarrow}}{4 \pi M_{s}} \frac{1}{d_{1}},
$$

where $\gamma$ is the gyromagnetic ratio, $M_{s}$ is the saturation magnetization, and $d_{1}$ is the thickness of the ferromagnetic layer

${ }^{a)}$ Electronic mail: bheinric@sfu.ca
FM1. The inverse dependence of the Gilbert damping on the film thickness clearly testifies to its interfacial origin. In this case the layer FM1 acts as a spin pump and the layer FM2 acts as a spin sink. The spin pump and spin sink effects have been thoroughly quantitatively studied in $\mathrm{Au} / \mathrm{Fe} / \mathrm{Au} / \mathrm{Fe} / \mathrm{GaAs}(001)$ structures, see, e.g., Refs. 7 and 8 . The quantitative comparison with spin pumping theory is very good. ${ }^{8}$ The strength of spin pumping at RT was found to be only $14 \%$ lower than that predicted by theory, and it was in excellent agreement at He temperatures. This is an important result. In magnetic double layer structures spin dynamics studies can be carried out with a perfect spin sink, allowing one to determine the full strength of spin pumping.

The spin pump effect can also be observed in single FM films surrounded by NM layers, provided that the pumped spin current is transported away from the FM/NM interface. Interface damping was studied in NM/Py/NM sandwiches by Mizukami et al., ${ }^{9}$ where $\mathrm{NM}=\mathrm{Pt}, \mathrm{Pd}, \mathrm{Ta}$, and $\mathrm{Cu}$. The $\mathrm{NM}$ layers were $5 \mathrm{~nm}$ thick. No interface damping was observed with the $\mathrm{Ta}$ and $\mathrm{Cu}$ layers. Tserkovnyak et al. explained the lack of interface damping in $(\mathrm{Ta}, \mathrm{Cu}) / \mathrm{Py} /(\mathrm{Cu}, \mathrm{Ta})$ structures by long spin-diffusion lengths in $\mathrm{Cu}$ and $\mathrm{Ta}$. The 5-nm-thick $\mathrm{Cu}$ and $\mathrm{Ta}$ do not provide effective spin sinks. However, a substantial interface damping was observed in both the Pt and Pd layers. The results by Mizukami et al. were obtained on samples prepared by sputtering. Since Pd and Pt have a strong tendency to intermix with $3 d$ transition elements it is interesting to compare the results obtained from samples prepared by sputtering with samples prepared by molecularbeam epitaxy (MBE) techniques. The purpose of this paper is to study the spin pump effect in Pd overlayers using crystalline epitaxial $\mathrm{Pd} / \mathrm{Fe}(001)$ structures which were prepared by $\mathrm{MBE}$, where the intermixing between the $\mathrm{Fe}$ and $\mathrm{Pd}$ is known to be minimal. ${ }^{10}$

\section{SAMPLE GROWTH AND FMR MEASUREMENTS}

Metallic $n \mathrm{Pd} / 16 \mathrm{Fe}(001)$ films were grown on GaAs(001) by MBE using epi-ready $\operatorname{GaAs}(001)$ semi- 


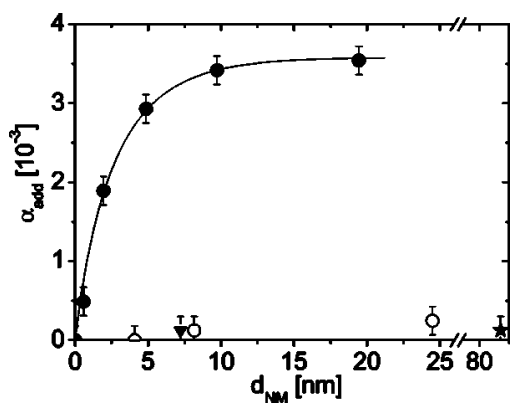

FIG. 1. The additional Gilbert damping $\alpha_{\text {add }}$ arising from spin pumping as a function of the Pd film thickness. The Pd data are shown in $(\mathbf{)})$. For comparison several points are shown for $\mathrm{Au}(\bigcirc), \mathrm{Ag}(\boldsymbol{\nabla})$, and $\mathrm{Cu}(\star)$. The solid line was obtained by fitting the Pd data using Eq. (8). $\lambda_{\mathrm{dec}}$ was found to be $9 \mathrm{~nm}$. $1 \mathrm{ML}$ of $\mathrm{Pd}$ corresponds to $0.2 \mathrm{~nm}$.

insulating templates, see details in Ref. 11. $n$ was between 3 and 200. The integers represent the number of monolayers (ML). All films were covered with Au for protection in ambient conditions. Pd has a lattice mismatch of $4.4 \%$ with respect to $\mathrm{Fe}$ and $4.9 \%$ with respect to $\mathrm{Au}$, and therefore samples with a sufficient thickness of Pd are affected by the relaxation of lattice strain. The presence of a self-assembled network of misfit dislocation half loops was observed by plan view transmission electron microscopy (TEM). ${ }^{12}$ Above a Pd thickness of $100 \mathrm{ML}$ the network of self-assembled misfit dislocations leads to strong two magnon scattering. Therefore, the study of intrinsic damping had to be carried out for the Pd films thinner than $100 \mathrm{ML}(20 \mathrm{~nm}) .^{12}$ The damping was investigated by ferromagnetic resonance (FMR) at 24 and $36 \mathrm{GHz}$.

\section{RESULTS AND DISCUSSION}

The role of $\mathrm{Pd}$ in the propagation of a spin current was investigated by monitoring the FMR linewidth as a function of the Pd overlayer thickness in $n \mathrm{Pd} / 16 \mathrm{Fe} / \mathrm{GaAs}(001)$ structures. The FMR linewidth has two contributions: (a) the intrinsic Gilbert contribution corresponding to the Fe film bulk damping and (b) the contribution from the pumped spin current, which is dissipated in the Pd layer and thus contributes to the Fe interface damping. The additional Gilbert damping arising from spin pumping is shown in Fig. 1.

For comparison some results with $\mathrm{Au}, \mathrm{Ag}$, and $\mathrm{Cu}$ overlayers are shown. Clearly, $\mathrm{Pd}$ is different than the noble metals. For the Pd layers thicker than $10 \mathrm{~nm}(50 \mathrm{ML})$ the additional Gilbert damping saturates, i.e., Pd acts as a perfect spin sink. For the $\mathrm{Au}, \mathrm{Ag}$, and $\mathrm{Cu}$ overlayers the contribution from spin pumping remains so small that it is within the accuracy of our FMR measurements. $\mathrm{Au}, \mathrm{Ag}$, and $\mathrm{Cu}$ in this thickness range behave as spin accumulators, not spin sinks. Spin pumping from the Fe layer increases the spin momentum in $\mathrm{Cu}, \mathrm{Ag}$, and $\mathrm{Au}$, and the resulting backflow of spin current nearly compensates the spin pumping, resulting in a zero interface current and a negligible additional damping.

In order to discuss the spin pumping contribution in $\mathrm{Pd}$ it is informative to first estimate the momentum electron mean free path. The sheet resistance of the two samples $20 \mathrm{Au} / 50 \mathrm{Pd} / 16 \mathrm{Fe} / \mathrm{GaAs}(001)$ and $20 \mathrm{Au} / 16 \mathrm{Fe} / \mathrm{GaAs}(001)$ was measured by means of the van der Pauw technique. The sheet resistances were found to be $9.6 \Omega / \square$ and $19.9 \Omega / \square$. Since the Pd layer contributes in parallel to the overall sheet resistance one can conclude that the sheet resistance for the 50-ML-thick Pd film is approximately $18.7 \Omega / \square$. This sheet resistance leads to the resistivity $\varrho=18.2 \mu \Omega \mathrm{cm}$. This value is about two times bigger than that of bulk $\mathrm{Pd}, \rho_{\mathrm{Pd}}^{\text {bulk }}$ $=10.8 \mu \Omega \mathrm{cm} .{ }^{13}$ The measured resistivity allows one to estimate the mean free path $\lambda_{m}$ using a simple formula (valid only for a spherical Fermi surface)

$$
\frac{1}{\rho}=\frac{e^{2} N \lambda_{m}}{m * v_{F}}
$$

where $e$ is the elementary charge, $m^{*}$ is the electron effective mass, $N$ is the density of electrons, and $v_{F}$ is the Fermi velocity. The number of conduction electrons ( $\Gamma$-centered electron sheet) per Pd atom was found to be $0.37 .^{14}$ This results in the carrier density $N=2 \times 10^{15} \mathrm{~cm}^{-3}$. The effective mass of the conduction electrons is $m * \sim 2 m$, where $m$ is the freeelectron mass and the Fermi velocity is $v_{F}=5.6 \times 10^{7} \mathrm{~cm} / \mathrm{s}$. ${ }^{14}$ This results in $\lambda_{m} \simeq 9 \mathrm{~nm}$. One should realize that the sheet resistance in the $\mathrm{Pd}$ thin film is mostly determined by diffuse scattering at the interfaces. Therefore, $\lambda_{m}=9 \mathrm{~nm}$ significantly underestimates the mean free path inside the $\mathrm{Pd}$ film. A similar behavior was found for the $\mathrm{Au}$ thin films grown on Fe/GaAs(001). ${ }^{15}$ Since the momentum mean free path in our Pd overlayers is larger than the film thicknesses the spin-diffusion theory ${ }^{16}$ is not applicable in the interpretation of our results.

We will demonstrate below that the mean free spin decoherence length is less than the momentum mean free path in our Pd samples. In this limit, the spin flow pumped by the Fe layer is gradually attenuated in Pd before the momentum of the electron is changed. The spin flow pumped by Fe into $\mathrm{Pd}$ decays, preventing the net pumped spin momentum from returning back to the Fe film after reflection at the outer Pd interface. When the thickness of the sample is less than the mean free path, the backflow of the spin current can thus be described by

$$
\mathbf{I}_{s}^{\text {back }}=\mathbf{I}_{s}^{\text {pump }} e^{-2 d_{\mathrm{Pd}}^{\text {eff }} / \lambda_{\mathrm{dec}}},
$$

where $d_{\mathrm{Pd}}^{\text {eff }}$ is the effective thickness of the Pd film and $\lambda_{\mathrm{dec}}$ is the mean decoherence length. $\mathbf{I}_{s}^{\text {pump }}$ is given by Eq. (1). The factor 2 in the exponent appears because the effective thickness of the Pd film for the spin current making it back to $\mathrm{Fe}$ is twice the film thickness. The effective Pd film thickness $d_{\mathrm{Pd}}^{\text {eff }}$ is larger than $d_{\mathrm{Pd}}$. The ratio $d_{\mathrm{Pd}}^{\text {eff }} / \lambda_{\mathrm{dec}}$ can be estimated by including the length of the electron path propagating under an angle $\theta$ with respect to the film normal. This calculation includes only electrons at the Fermi surface participating in spin pumping. For a spherical Fermi surface one can write

$$
e^{-2 d_{\mathrm{Pd}}^{\mathrm{eff}} / \lambda_{\mathrm{dec}}}=\frac{1}{\pi k_{F}^{2}} \int_{0}^{k_{F}} 2 \pi k_{\|} d k_{\|} e^{-2 d_{\mathrm{Pd}} / \cos \theta \lambda_{\mathrm{dec}}},
$$

where $k_{\|}$is the component of the $k$ vector parallel to the interface and $\cos \theta=\left[1-\left(k_{\|} / k_{F}\right)^{2}\right]^{0.5}$. The net spin current across the interface is then given by 


$$
\mathbf{I}_{s}=\mathbf{I}_{s}^{\mathrm{pump}}\left(1-e^{-2 d_{\mathrm{Pd}}^{\mathrm{eff}} / \lambda_{\mathrm{dec}}}\right),
$$

which leads to the enhanced Gilbert damping due to spin pumping

$$
\alpha_{\mathrm{Pd}}=\frac{\gamma \hbar g^{\uparrow \downarrow}}{4 \pi M_{s} d_{\mathrm{Fe}}}\left(1-e^{-2 d_{\mathrm{Pd}}^{\mathrm{eff}} / \lambda_{\mathrm{dec}}}\right) .
$$

The thickness dependence in Fig. 1 can be fit by two independent parameters, $g^{\uparrow \downarrow}$ and $\lambda_{\mathrm{dec}}$. The resulting parameters are $g^{\uparrow \downarrow}=0.9 \times 10^{15} \mathrm{~cm}^{-2}$ and $\lambda_{\mathrm{dec}}=9 \mathrm{~nm}$. When the $\mathrm{Pd}$ layer is thicker than the momentum mean free path, there might still be a backflow of electron $I_{s}^{\mathrm{back}} / I_{s}^{\mathrm{pump}}$ $\sim \exp \left(-2 \lambda_{m} / \lambda_{\text {dec }}\right)$. For $\lambda_{m}=\lambda_{\text {dec }}$ gives $I_{s}^{\text {back }} / I_{s}^{\text {pump }} \sim 0.1$. The backflow would lead to a smaller value of the measured spin mixing conductance than that expected from the electron band calculations. Realizing that the mean free path inside the Pd layer is larger than the Pd layer thickness this correction is small in our samples.

From Eq. (2) one can estimate the spin mixing conductance, $\quad g^{\uparrow \downarrow}=0.5$ and $0.7 \times 10^{15} \mathrm{~cm}^{-2}$ assuming $0.37 \Gamma$ electrons/atom ${ }^{14}$ and $0.55 s-p$ electrons/atom, ${ }^{17}$ respectively. This is in reasonable agreement with the experimentally required value of $0.9 \times 10^{15} \mathrm{~cm}^{-2}$ considering that the band structure of Pd is complex and Eq. (2) can be considered only a crude approximation. First-principles band calculations are required to account for complexity of the $\mathrm{Pd}$ band structure which can affect both the spin mixing conductance and Shavrin resistance. $\lambda_{\mathrm{dec}}$ is comparable to the momentum mean free path, $\lambda_{m}=9 \mathrm{~nm}$ obtained from the crude interpretation of the sheet resistance.

The mean free path of electrons in the Pd layer is larger, see above; this implies that the spin decoherence happens on the shorter length scale than the bulk momentum scattering. A good exponential fit in Fig. 1 suggests that the interface diffuse momentum scattering at the $\mathrm{Pd}$ /air interface does not affect the spin decoherence in Pd. The spin current is randomized mostly inside the Pd layer. Bulk Pd is known to have strong spin electron-electron correlation effects having a large Stoner enhancement factor resulting in enhanced paramagnetic susceptibility compared to $\mathrm{Ag}, \mathrm{Au}$, and $\mathrm{Cu}{ }^{18}$ Associated local fluctuating magnetic moments (paramagnons) are believed to make Pd suitable, under the right con- ditions, for establishing a long-range ferromagnetic state. ${ }^{19}$ One can envision that paramagnons in $\mathrm{Pd}$ can lead to an effective long-range decoherence of spin current. This means that the direction of the pumped spin momentum gets randomized by large spin fluctuations inside $\mathrm{Pd}$; and, consequently, the spin momentum backflow loses its net spin momentum and is unable to compensate the spin current generated by spin pumping. The spin mixing conductance in our samples is lower than that required to interpret the data by Mizukami et al. Their measurements require $g^{\uparrow \downarrow}=1.4$ $\times 10^{15} \mathrm{~cm}^{-2}$ for 5-nm-thick Pd. This is by a factor of 1.6 bigger than that observed in our studies. The difference between these two experiments can be due to the difference in sample preparation. The results by Mizukami et al. suggest that sputtering leads to an enhanced value of $g^{\uparrow \downarrow}$ and thus the intermixing of FM and $\mathrm{Pd}$ increases the strength of spin pumping.

${ }^{1}$ Y. Tserkovnyak, A. Brataas, and G. Bauer, Phys. Rev. Lett. 88, 117601 (2002).

${ }^{2}$ A. Brataas, Y. Nazarov, and G. Bauer, Eur. Phys. J. B 22, 99 (2001).

${ }^{3}$ A. Brataas, Y. Tserkovnyak, G. Bauer, and B. Halperin, Phys. Rev. B 66, 060404 (2002).

${ }^{4}$ K. Xia, P. Kelly, G. Bauer, A. Brataas, and I. Turek, Phys. Rev. B 65, 220401(R) (2002).

${ }^{5}$ A. Brataas, Y. V. Nazarov, and G. E. W. Bauer, Phys. Rev. Lett. 84, 2481 (2000).

${ }^{6}$ M. Stiles and A. Zangwill, Phys. Rev. B 66, 014407 (2002).

${ }^{7}$ B. Heinrich, Y. Tserkovnyak, G. Woltersdorf, A. Brataas, R. Urban, and G. Bauer, Phys. Rev. Lett. 90, 187601 (2003).

${ }^{8}$ B. Heinrich, G. Woltersdorf, R. Urban, and E. Šimanek, J. Appl. Phys. 93, 7545 (2003).

${ }^{9}$ S. Mizukami, Y. Ando, and T. Miyazaki, J. Magn. Magn. Mater. 226, 1640 (2001).

${ }^{10}$ E. Fullerton, S. Stoeffler, K. Ounadjela, B. Heinrich, J. Bland, and Z. Celinski, Phys. Rev. B 51, 6364 (1995).

${ }^{11}$ R. Urban, G. Woltersdorf, and B. Heinrich, Phys. Rev. Lett. 87, 217204 (2001).

${ }^{12}$ G. Woltersdorf and B. Heinrich, Phys. Rev. B 69, 184417 (2004).

${ }^{13}$ CRC Handbook of Materials (McGraw-Hill, New York, 1990).

${ }^{14}$ D. Dye, S. Campbell, G. Crabtree, J. Ketterson, N. Sandesara, and J. Vuillemin, Phys. Rev. B 23, 462 (1981).

${ }^{15}$ A. Enders, T. Monchesky, K. Myrtle, R. Urban, B. Heinrich, J. Kirschner, X.-G. Zhang, and W. Butler, J. Appl. Phys. 89, 7110 (2001).

${ }^{16}$ R. Urban, B. Heinrich, and G. Woltersdorf, J. Appl. Phys. 93, 8280 (2003).

${ }^{17}$ V. Moruzzi and P. Marcus, Phys. Rev. B 39, 471 (1989).

${ }^{18}$ M. Brodsky and A. Freeman, Phys. Rev. Lett. 45, 133 (1980).

${ }^{19}$ P. Larson, I. Mazin, and D. Singh, Phys. Rev. B 69, 064429 (2004). 